\title{
Atrial Rotor Modulation by Localized Dofetilide Application: An In Silico Study
}

\author{
Sandra Pérez-Buitrago ${ }^{1}$, Juan P Ugarte ${ }^{2}$, Catalina Tobón ${ }^{3}$ \\ ${ }^{1}$ GI2B, Instituto Tecnológico Metropolitano, Medellín, Colombia \\ ${ }^{2}$ GIMSC, Universidad de San Buenaventura, Medellín, Colombia \\ ${ }^{3}$ MATBIOM, Universidad de Medellín, Medellín, Colombia
}

\begin{abstract}
The rotor ablation has gained favor in recent years to treat chronic atrial fibrillation $(C A F)$. However, it may cause complications in patients and the results remain suboptimal. Besides, antiarrhythmic drugs for the management of AF are not sufficiently effective and can cause significant cardiac and extracardiac side effects.

To reduce the risk and increase the effectiveness, a rotor modulation via the localized release of dofetilide is proposed through an in silico study.

A computational model of two-dimensional atrial tissue was implemented using human cell model under CAF conditions. Dofetilide was modeled blocking the ionic currents $I_{K r}$ and $I_{K A C h}$ using Hill's equation. A CAF episode, sustained by a rotor, was simulated. Different concentrations of dofetilide were applied in specific distributions based on the rotor tip location, detected by phase map analysis.

Non-localized application of dofetilide resulted in the benefit of CAF progression. In contrast, localized dofetilide liberation terminated the CAF episode. These results could have therapeutic implications in novel treatments of $C A F$.
\end{abstract}

\section{Introduction}

Atrial fibrillation (AF) is a progressive pathology presenting from short and infrequent, to longer and recurrent episodes, becoming in many cases permanent or chronic [1]. Distinct approaches are applied for the AF management. Non-symptomatic patients are generally treated with anticoagulants and with a rate control strategy. For the symptomatic ones, antiarrhythmic drugs and catheter ablation to recover sinus rhythm are adopted. Nevertheless, the pharmacologic therapy efficacy remains poor and it can have serious side effects. Moreover, the ablation procedure still presents suboptimal outcomes [2].

The success rate of pulmonary vein ectopy ablation remains around $50 \%$ to $70 \%$ in paroxysmal $\mathrm{AF}$ patients, with lower success in CAF patients. This situation has motivated investigations looking for defining additional ablation targets, including stable rotors and fractionated atrial electrograms. Rotor ablation efficacy has been a topic of great debate due to the wide variability in success rates among clinical centres [2,3].

Dofetilide is indicated as a class III antiarrhythmic drug for $\mathrm{AF}$, but it may trigger the ventricular tachycardia torsade de pointes by the atrial action potential (APD) and QT interval prolongation. Therefore, dofetilide should be delivered in atrial specific sites without affecting the ventricles [4].

Bearing this idea in mind, the aim of this work is to propose and evaluate a therapy based on the rotor modulation via localized release of dofetilide. In a previous work, mathematical models of the dofetilide effect on human APD, $I_{K r}$ and $I_{K A C h}$ currents were assessed [5].

The proposed procedure seeks to improve the effectiveness of CAF therapy, by minimizing the side effects and by providing personalized patient care.

\section{Methodology}

\subsection{Electrophysiological model of human atrial cardiomyocytes}

The Courtemanche-Ramirez-Nattel-Kneller [6,7] membrane formalism to simulate the atrial action potential was implemented. A $0.005 u M$ of acetylcholine concentration was simulated.

The transmembrane voltage $\left(\mathrm{V}_{\mathrm{m}}\right)$ is calculated by the equation:

$$
C_{m} \frac{d V_{m}}{d t}+I_{i o n}+I_{\text {stim }}=0
$$

where $C_{m}$ is the specific membrane capacitance $(100 \mathrm{pF})$, $I_{\text {ion }}$ is the total transmembrane ionic current and $I_{\text {stim }}$ is the stimulus current.

To reproduce the electrical remodeling generated by $\mathrm{CAF}$, changes in conductance of specific ionic channels have been incorporated in the cell model according to experimental studies $[8,9]$. 


\subsection{Model of dofetilide effect on $I_{K r}$ and IKACh}

The steady state fraction of blocked channels $b$ was calculated in the presence of different concentrations of dofetilide using the Hill equation (2). This equation was used to develop a basic model of the dofetilide effect on the rapid component of the delayed rectifier potassium current $\mathrm{I}_{\mathrm{Kr}}$ and acetylcholine activated potassium current IKACh.

$$
b=\frac{1}{1+\left(\frac{I C_{50}}{D_{d}}\right)^{h}},
$$

where $\mathrm{IC}_{50}$ is the half maximal inhibitory concentration for the current blockade due to dofetilide and $D_{d}$ is the dofetilide concentration. The applied value of $\mathrm{IC}_{50}$ related with $\mathrm{I}_{\mathrm{Kr}}$ was $0.32 \mu \mathrm{M}$, found in HERG $\mathrm{K}+$ channels [9]. A value of $6.6 \mu \mathrm{M}$ for $\mathrm{IC}_{50}$ was used in order to block $\mathrm{I}_{\mathrm{KACh}}$ and it agrees with reported concentrations for isolated atrial myocytes [10]. The value of the Hill coefficient $h$ was set to 1 .

\subsection{D model of human atrial tissue and electrical propagation}

A 2D model of human atrial tissue was implemented in MATLAB under CAF conditions, it consists of a $4 \times 4 \mathrm{~cm}$ matrix, discretized at a spatial resolution of $400 \mu \mathrm{m}$, to form a mesh of $100 \times 100$ nodes.

The electrical propagation of action potential over the tissue was described by the reaction-diffusion equation (3):

$$
\frac{1}{S_{v}} \nabla \cdot\left(D \nabla V_{m}\right)=C_{m} \frac{\delta V_{m}}{\delta t}+I_{\text {ion }}+I_{s t i m},
$$

where $\mathrm{S}_{\mathrm{v}}$ is the surface/volume ratio and $D$ is the conductivity tensor. The equation (3) was solved using a semi-spectral numerical scheme [11].

A CAF episode sustained by a rotor was generated implementing a S1-S2 cross-field stimulation protocol. The S1 stimulus was applied at the left boundary of the tissue. The $\mathrm{S} 2$ stimulus is rectangular $(2 \times 2 \mathrm{~cm})$ and was applied to a corner of the domain [12]. The approximate volume for a single cardiomyocyte is $2.16 \times 10^{-5} \mu 1$. Thus, the total volume for the 10.000 nodes is $20.1 \mu$ l.

\subsection{Patterns of dofetilide release}

In order to assess the effect of dofetilide application on the CAF episode, the following delivery configurations were tested. A homogeneous distribution over the entire tissue at concentrations between $1 \mu \mathrm{M}$ and $500 \mu \mathrm{M}$. Localized dofetilide application was designed in geometrical patterns defined with respect to the rotor tip location, at concentrations varying from $1 \mu \mathrm{M}$ to $20 \mu \mathrm{M}$.

For this purpose, the phase maps were calculated and the rotor tip was defined as the point where all phases between $-\pi$ and $\pi$ converge [13]. The designed dofetilide liberation patterns are as follows: simple ring (Figure 1a) and double ring (Figure 1b), both enclosing all the points of the rotor tip trajectory; and a horizontal rectangle, composed by $11 \times 100$ nodes, that crosses trough the rotor tip path (Figure 1c).

A

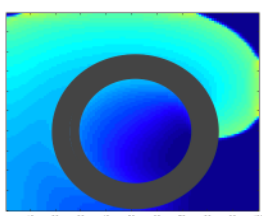
B

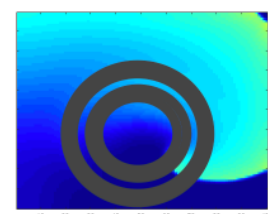

C

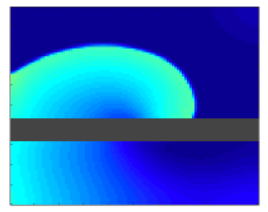

Figure 1. Patterns for dofetilide application (a) ring, (b) double ring, (c) rectangle.

\section{Results and discussion}

\subsection{D simulation of chronic atrial fibrillation}

The stable clockwise rotor generated in the 2D model after applying the S1-S2 protocol is shown in Figure 2a, and the corresponding tip trajectory is presented in Figure $2 b$.

Figures $2 \mathrm{c}$ and $2 \mathrm{~d}$ show the rotors after $10 \mathrm{~s}$ of applying $500 \mu \mathrm{M}$ of dofetilide to the whole tissue. These results suggest that when dofetilide is globally applied, independently of the concentration, rotor becomes more stable which could benefit the progression of AF [14].

By implementing the simple ring pattern, the rotor becomes a macro-reentry that turns around an unexcitable region caused by the dofetilide. Such behaviour is similar to a fibrotic or ischemic zone [14]. The simulation was carried out for $6 \mathrm{~s}$ and the macro-reentry persisted. Figure 2e shows the rotor at the end of the simulation and Figure $2 \mathrm{f}$ depicts the macro-reentry described from the tip tracking.

On the other hand, the dofetilide liberation in a double ring pattern did not terminate the rotor activity (figure $2 \mathrm{~g}$ ) and it can be observed also in the rotor tip trajectory generated (figure $2 \mathrm{~h}$ ).

Finally, the dofetilide was applied as the rectangle pattern that crosses the rotor tip center. Figures 3a-c and Figures 3d-f present the outcomes after $2 \mathrm{~s}$ and $4 \mathrm{~s}$ of simulation. In both cases, the rotor activity ends at different concentrations. The rotor tip trajectory, depicted in Figure 4 , evinces that this localized dofetilide application destabilizes the rotor, generating a meandering trajectory until its termination. 
A

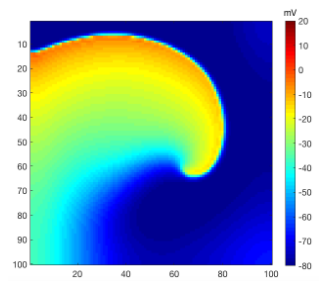

C

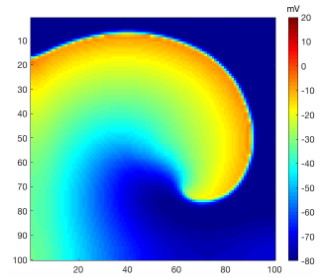

$\mathbf{E}$

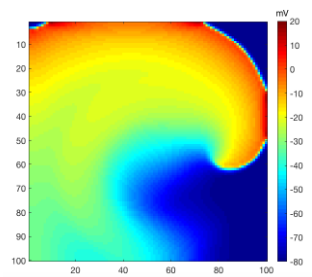

G

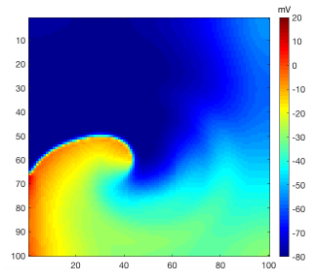

B

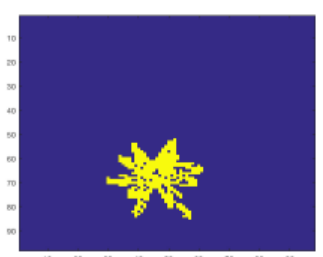

D

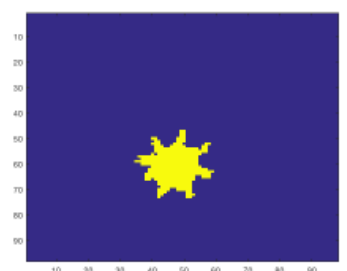

$\mathbf{F}$

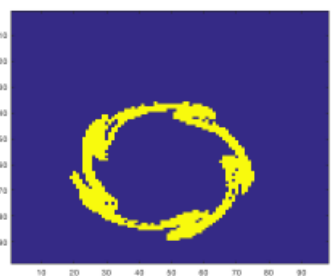

H

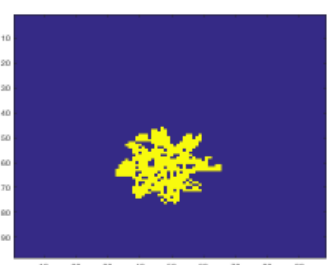

Figure 2. Rotor and tip trajectory simulations after $10 \mathrm{~s}$ of applying: $0 \mu \mathrm{M}$ of dofetilide (a,b), and $50 \mu \mathrm{M}$ of dofetilide to the whole tissue (c,d), as the ring pattern $(\mathrm{e}, \mathrm{f})$, and as the double ring pattern $(\mathrm{g}, \mathrm{h})$.

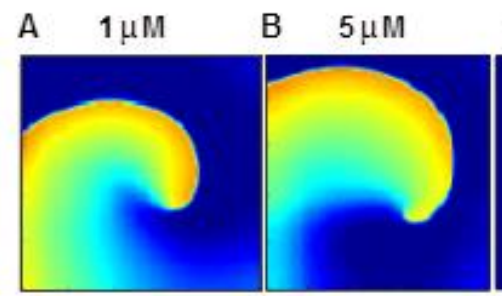

C $\quad 10-20 \mu \mathrm{M}$

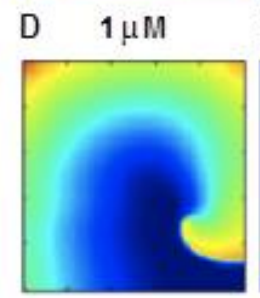

E $\quad 5 \mu \mathrm{M}$
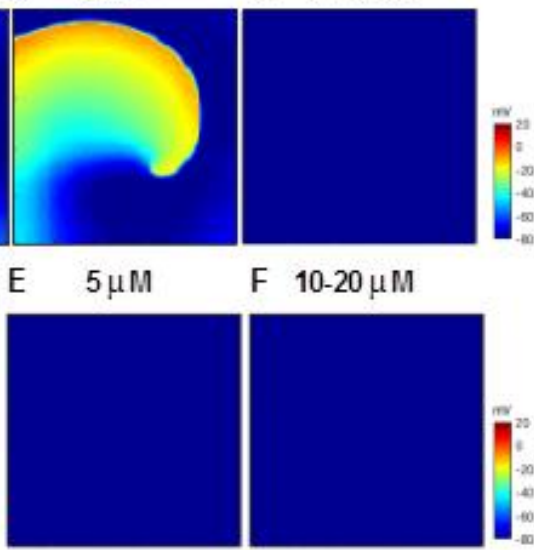

F $10-20 \mu \mathrm{M}$

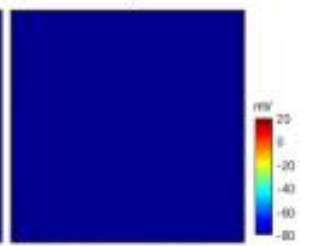

Figure 3. Rotor dynamics after applying dofetilide under a rectangular pattern at three distinct concentrations after (a-c) $2 \mathrm{~s}$ and (d-f) $7 \mathrm{~s}$ of simulation.

The minimum concentration of dofetilide, yielding rotor termination after $4 \mathrm{~s}$ of its applications, is $5 \mu \mathrm{M}$. Therefore, for the tissue volume of $20.1 \mathrm{ml}$ considered in this work, a single dose of $44.4 \mathrm{ng}$ would be required. This dosage is significantly low compared to clinically recommended oral doses, ranging from $125 \mathrm{mg}$ to $2500 \mathrm{mg}$ per day [15-17].

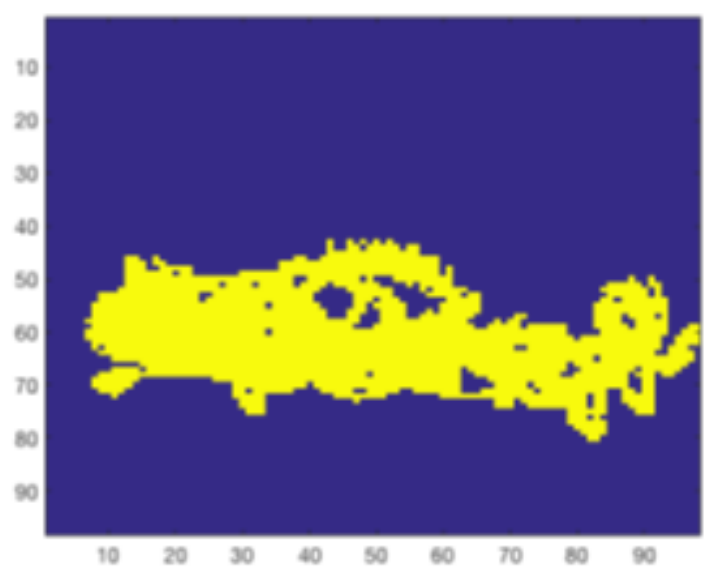

Figure 4. Rotor tip trajectory after applying $5 \mu \mathrm{M}$ of dofetilide under a rectangular pattern crossing the rotor tip.

\section{Conclusions}

A possible proarrhythmic effect was detected when applying dofetilide homogeneously to the whole tissue in the $2 \mathrm{D}$ model.

The results obtained indicate that it is possible to terminate the rotor activity by applying $5 \mu \mathrm{M}$ of dofetilide during $4 \mathrm{~s}$ in a localized form and following a rectangular pattern of $11 \times 100$ elements that crosses the tip of the rotor.

Possible implications of the proposed therapy would include an important reduction of CAF treatment costs, since it would be possible to end the rotor activity with a single dose of dofetilide in the range of nanograms in contrast to the prolonged oral treatments. As a future work, systematic experimental test of this approach, with cellular and animal models, are needed to advance in the development of an optimal AF treatment.

\section{Acknowledgements}

The financial support from the Departamento administrativo de ciencia, tecnología e innovación de la República de Colombia COLCIENCIAS, under project FP44842-075-2015 (2014-0401), is gratefully acknowledged.

\section{References}


[1] D. H. Lau, D. Linz, U. Schotten, R. Mahajan, P. Sanders, and J. M. Kalman, "Pathophysiology of paroxysmal and persistent atrial fibrillation: rotors, foci and fibrosis," Hear. Lung Circ., vol. 9506, no. 17, pp. 1-7, 2017.

[2] D. E. Krummen, V. Swarup, and S. M. Narayan, "The role of rotors in atrial fibrillation," J. Thorac. Dis., vol. 7, no. 2, pp. 142-151, Feb. 2015.

[3] R. Parameswaran, A. Voskoboinik, A. Gorelik, G. Lee, P. M. Kistler, P. Sanders, and J. M. Kalman, "Clinical impact of rotor ablation in atrial fibrillation: a systematic review," EP Eur., vol. 20, no. 7, pp. 1099-1106, Jul. 2018.

[4] U. Ravens and K. E. Odening, "Atrial fibrillation: Therapeutic potential of atrial $\mathrm{K}+$ channel blockers," Pharmacol. Ther., vol. 176, pp. 13-21, Aug. 2017.

[5] C. Tobón, S. Pérez, J. P. Ugarte, and J. Saiz, "Dofetilide effect on human atrial action potential under normal and atrial fibrillation conditions. In silico study BT - VII Latin American Congress on Biomedical Engineering CLAIB 2016, Bucaramanga, Santander, Colombia, October 26th -28th, 2016," 2017, pp. 38-41.

[6] M. Courtemanche, R. J. Ramirez, and S. Nattel, "Ionic mechanisms underlying human atrial action potential properties: insights from a mathematical model.," Am. J. Physiol., vol. 275, no. 1 Pt 2, pp. H301-21, Jul. 1998.

[7] M. Courtemanche, R. J. Ramirez, and S. Nattel, "Ionic targets for drug therapy and atrial fibrillation-induced electrical remodeling: insights from a mathematical model.," Cardiovasc. Res., vol. 42, no. 2, pp. 477-489, May 1999.

[8] D. R. Van Wagoner, "Electrophysiological remodeling in human atrial fibrillation.," Pacing Clin. Electrophysiol., vol. 26, no. 7 Pt 2, pp. 1572-1575, 2003.

[9] E. Ficker, W. Jarolimek, J. Kiehn, A. Baumann, and A. M. Brown, "Molecular Determinants of Dofetilide Block of HERG K \% Channels," pp. 386-395, 2015.

[10] N. Voigt, N. Rozmaritsa, A. Trausch, T. Zimniak, T. Christ, E. Wettwer, K. Matschke, D. Dobrev, and U. Ravens, "Inhibition of IK,ACh current may contribute to clinical efficacy of class i and class III antiarrhythmic drugs in patients with atrial fibrillation," Naunyn. Schmiedebergs. Arch. Pharmacol., vol. 381, no. 3, pp. 251-259, 2010.

[11] A. Bueno-Orovio, D. Kay, and K. Burrage, "Fourier spectral methods for fractional-in-space reactiondiffusion equations," BIT Numer. Math., vol. 54, no. 4, pp. 937-954, 2014.

[12] O. Berenfeld, "The major role of IK1 in mechanisms of rotor drift in the atria: a computational study," Clin. Med. Insights Cardiol., vol. 10, pp. 71-79, 2016.

[13] J. P. Ugarte, C. Tobón, A. Orozco-Duque, M. A. Becerra, and J. Bustamante, "Effect of the electrograms density in detecting and ablating the tip of the rotor during chronic atrial fibrillation: An in silico study," Europace, vol. 17, pp. ii97-ii104, 2015.

[14] M. Guillem, A. M. Climent, M. Rodrigo, F. FernandezAviles, F. Atienza, and O. Berenfeld, "Presence and stability of rotors in atrial fibrillation: Evidence and therapeutic implications," Cardiovasc. Res., vol. 109, no. 4, pp. 480-492, 2016.

[15] S. Singh, R. G. Zoble, L. Yellen, M. a Brodsky, G. K.
Feld, M. Berk, and C. B. Billing, "Efficacy and safety of oral dofetilide in converting to and maintaining sinus rhythm in patients with chronic atrial fibrillation or atrial flutter: the symptomatic atrial fibrillation investigative research on dofetilide (SAFIRE-D) study.," Circulation, vol. 102, no. 19, pp. 2385-2390, 2000.

[16] M. J. Allen, D. J. Nichols, and S. D. Oliver, "The pharmacokinetics and pharmacodynamics of oral dofetilide after twice daily and three times daily dosing," Clin. Pharmacol., vol. 50, pp. 247-253, 2000.

[17] A. Jaiswal and S. Goldbarg, "Dofetilide induced torsade de pointes: Mechanism, risk factors and management strategies," Indian Heart J., vol. 66, no. 6, pp. 640-648, 2014.

Address for correspondence.

Sandra Milena Pérez Buitrago

Calle 73 No. 76A - 354, ZIP code 050034. Medellìn-Colombia. sandraperez@itm.edu.co 